\title{
Mean curvature flows and isotopy problems
}

\author{
Mu-Tao Wang
}

\begin{abstract}
In this note, we discuss the mean curvature flow of graphs of maps between Riemannian manifolds. Special emphasis will be placed on estimates of the flow as a non-linear parabolic system of differential equations. Several global existence theorems and applications to isotopy problems in geometry and topology will be presented. The results are based on joint works of the author with his collaborators I. Medoš, K. Smoczyk, and M.-P. Tsui.
\end{abstract}

\section{Introduction}

We start with classical minimal surfaces in $\mathbb{R}^{3}$ (see for example $[\mathbf{2 6}]$ ). Suppose a surface $\Sigma$ is given as the graph of a function $f=f(x, y)$ over a domain $\Omega \subset \mathbb{R}^{2}$ :

$$
\Sigma=\{(x, y, f(x, y)) \mid(x, y) \in \Omega\} .
$$

The area $A(\Sigma)$ is given by the formula

$$
A(\Sigma)=\int_{\Omega} \sqrt{1+|\nabla f|^{2}}
$$

The Euler-Lagrange equation for the area functional is derived to be

$$
\operatorname{div}\left(\frac{\nabla f}{\sqrt{1+|\nabla f|^{2} \mid}}\right)=0
$$

Equation (1.1), so called the minimal surface equation, is one of the most studied nonlinear elliptic PDE and there are many beautiful classical results such as the celebrated Bernstein's conjecture for entire solutions $[\mathbf{3}, \mathbf{4}]$. The Dirichlet problem is uniquely solvable as long as the mean curvature of the boundary $\partial \Omega$ is positive [20]. In addition, any Lipschitz solution is smooth and analytic $[\mathbf{2 4}, \mathbf{2 3}]$.

The author was partially supported by the National Science Foundation under grant DMS-1105483. He would like to thank his collaborators I. Medoš, K. Smoczyk, and M.-P. Tsui. 
The corresponding parabolic equation is called the mean curvature flow. Here we have a time-dependent surface $\Sigma_{t}$, given as the graph of a function $f=f(x, y, t)$ for each $t$, and $f$ satisfies

$$
\frac{\partial f}{\partial t}=\sqrt{1+|\nabla f|^{2}} \operatorname{div}\left(\frac{\nabla f}{\sqrt{1+|\nabla f|^{2}}}\right)
$$

This is the negative gradient flow of the area functional. In fact, the normal component of the velocity vector of the graph of $f(x, y, t)$ in $\mathbb{R}^{3}$ is exactly the mean curvature vector.

The equation has been extensively studied by many authors such as Huisken $[\mathbf{1 5}, \mathbf{1 6}]$, Ecker-Huisken [9, 10], Ilmanen [19], Andrews [1], White $[\mathbf{4 1}, \mathbf{4 2}]$, Huisken-Sinestrari, $[\mathbf{1 7}, \mathbf{1 8}]$ X.-J. Wang [40], Colding-Minicozzi [8], etc. Note that though the elliptic equation (1.1) is in divergence form, the parabolic equation is not. Therefore, standard results from parabolic PDE theory do not readily apply.

We can also consider the equations in parametric form. Suppose the surface is given by an embedding: $\vec{X}(u, v)=\left(X_{1}(u, v), X_{2}(u, v), X_{3}(u, v)\right) \in$ $\mathbb{R}^{3}$. The minimal surface equation (1.1) is equivalent to

$$
\left(\Delta_{\Sigma} X_{1}, \Delta_{\Sigma} X_{2}, \Delta_{\Sigma} X_{3}\right)=(0,0,0)
$$

where $\Sigma$ is the image surface of $\vec{X}$ and $\Delta_{\Sigma}$ is the Laplace operator with respect to the induced metric on $\Sigma$. In fact, $\vec{H}=\Delta_{\Sigma} \vec{X}$ is the mean curvature vector of $\Sigma$. However, this elegant form has a disadvantage that it is invariant under reparametrization and thus represents a degenerate elliptic system for $\left(X_{1}, X_{2}, X_{3}\right)$. The same phenomenon is encountered for any curvature equation in which the diffeomorphism group appears as the symmetry group.

The corresponding parabolic equation for a family of time-dependent embeddings $\vec{X}(u, v, t)$ is

$$
\frac{\partial \vec{X}}{\partial t}=\Delta_{\Sigma} \vec{X}
$$

For this, the mean curvature flow is often referred as the heat equation for submanifolds, just as the Ricci flow is the heat equation for Riemannian metrics. However, it is clear that the equation is of nonlinear nature as $\Delta_{\Sigma}$ depends on first derivatives of $\vec{X}$.

Our subject of study in this note is a submanifold of "higher codimension", such as a 2-surface in a 4-dimensional space given by the graph of a vector value function $(f, g)$ :

$$
\Sigma=\{(x, y, f(x, y), g(x, y)) \mid(x, y) \in \Omega\} .
$$

The area of $\Sigma$ is then

$$
A(\Sigma)=\int_{\Omega} \sqrt{1+|\nabla f|^{2}+|\nabla g|^{2}+\left(f_{x} g_{y}-f_{y} g_{x}\right)^{2}}
$$

and the Euler-Lagrange equation is a non-linear elliptic system for $f$ and $g$ (see the next paragraph). 
In general, we consider a vector-valued function $\vec{f}: \Omega \subset \mathbb{R}^{n} \rightarrow \mathbb{R}^{m}$ and $\Sigma$ is the graph of $\vec{f}=\left(f^{1}, \cdots, f^{m}\right)$ in $\mathbb{R}^{n+m}$. Denote the induced metric on $\Sigma$ by

$$
g_{i j}=\delta_{i j}+\sum_{\alpha=1}^{m} \frac{\partial f^{\alpha}}{\partial x^{i}} \frac{\partial f^{\alpha}}{\partial x^{j}} .
$$

The volume of $\Sigma$ is

$$
\int_{\Omega} \sqrt{\operatorname{det} g_{i j}}
$$

and the Euler-Lagrange equation, which is often referred as the minimal surface system, is

$$
\sum_{i, j=1}^{n} g^{i j} \frac{\partial^{2} f^{\alpha}}{\partial x^{i} \partial x^{j}}=0, \alpha=1, \cdots, m,
$$

where $g^{i j}=\left(g_{i j}\right)^{-1}$ is the inverse of $g_{i j}$.

The corresponding parabolic equation is the mean curvature flow

$$
\frac{\partial f^{\alpha}}{\partial t}=\sum_{i, j=1}^{n} g^{i j} \frac{\partial^{2} f^{\alpha}}{\partial x^{i} \partial x^{j}}, \alpha=1, \cdots, m .
$$

There is no reason to stop there and we can consider the even more general situation when $\mathbf{f}: M_{1} \rightarrow M_{2}$ is a differentiable map between Riemannian manifolds, and $\Sigma$ is the graph of $\mathbf{f}$ in $M_{1} \times M_{2}$ for $M_{1}$ an $n$-dimensional Riemannian manifold and $M_{2}$ an $m$-dimensional one.

In contrast to the codimensional one case, in an article by LawsonOsserman [21] entitled "Non-existence, non-uniqueness and irregularity of solutions to the minimal surface system", the undesirable features of the system mentioned in the title are demonstrated. The codimension one case, i.e. $m=1$, is essentially a scalar equation. In addition, the normal bundle of an oriented hypersurface is always trivial. On the other hand, $m>1$ corresponds to a genuine systems and the components $f^{1}, \cdots, f^{m}$ interact with each other. Moreover, the geometry of normal bundle can be rather complicated.

Nevertheless, we managed to obtain estimates and prove several global existence theorems for higher-codimensional mean curvature flows with appropriate initial data. I shall discuss the methods in the next section before presenting the results.

\section{Method of proofs}

Let us start with the $C^{1}$ estimate. In the codimension-one case (see [9] for the equation in a slightly different but equivalent form), $m=1$, an important equation satisfied by $J_{1}=\frac{1}{\sqrt{1+|\nabla f|^{2}}}$ is

$$
\frac{d}{d t} J_{1}=\Delta_{\Sigma} J_{1}+R_{1}\left(\nabla f, \nabla^{2} f\right) .
$$


The term $R_{1}>0$ is quadratic in $\nabla^{2} f$.

Let us look at the $m=2, n=2$ case. We can similarly take

$$
J_{2}=\frac{1}{\sqrt{1+|\nabla f|^{2}+|\nabla g|^{2}+\left(f_{x} g_{y}-f_{y} g_{x}\right)^{2}}}
$$

and compute the evolution equation:

$$
\frac{d}{d t} J_{2}=\Delta_{\Sigma} J_{2}+R_{2}\left(\nabla f, \nabla g, \nabla^{2} f, \nabla^{2} g\right) .
$$

It is observed that $R_{2}$ is quadratic in $\nabla^{2} f$ and $\nabla^{2} g$ and is positive if $\left|f_{x} g_{y}-f_{y} g_{x}\right| \leq 1$ (The is can be found in [33], though in a somewhat more complicated form).

A natural idea is to investigate how the quantity $f_{x} g_{y}-f_{y} g_{x}$, or the Jacobian of the map $(f, g)$ changes along the flow. Together with the maximal principle, it was shown in $[\mathbf{3 2}, \mathbf{3 3}]$ that:

(1) $f_{x} g_{y}-f_{y} g_{x}=1$ is "preserved" along the mean curvature flow (area preserving).

(2) $\left|f_{x} g_{y}-f_{y} g_{x}\right|<1$ is "preserved" along the mean curvature flow (area decreasing).

Here a condition is "preserved" means if the condition holds initially, it remains true later as long as the flow exists smoothly.

Combining with the evolution equation of $J_{2}$ and applying the maximum principle again show that $J_{2}$ has a lower bound, which in turn gives a $C^{1}$ estimate of $f$ and $g$. Notice that $J_{2}$ can be regarded as the Jacobian of the projection map onto the first factor of $\mathbb{R}^{2}$. Thus by the inverse function theorem, the graphical condition is also preserved.

Such a condition indeed corresponds to the Gauss map of the submanifold lies in a totally geodesic or geodesically convex subset of the Grassmannian [36]. The underlying fact for this calculation is based on the observation [36] that the Gauss map of the mean curvature flow is a (nonlinear) harmonic map heat flow.

In codimension one case, the higher derivatives estimates follows from the $C^{1}$ estimates [9]. The elliptic analogue is the theorem of Moser which states that any Lipschitz solution of the minimal surface equation is smooth. The scenario is totally different in the higher codimension case. LawsonOsserman constructed minimal cones in higher codimensions and thus a Lipschitz solution to the minimal surface system with $m>1$ may not be smooth at all.

Here we use "blow-up analysis" for geometric evolution equations. An important tool is Huisken-White's monotonicity formula $[\mathbf{1 5}, \mathbf{4 1}]$ which characterizes central blow-up profiles as solutions of the elliptic equation:

$$
\vec{H}=-\vec{X} \text {. }
$$

In general, singularity profiles for parabolic equations are soliton (selfsimilar) solutions of the equation. In the case of mean curvature flows, soliton (self-similar) solutions are moved by homothety or translations of 
the ambient space. Exclusion of self-similar "area-preserving" or "areadecreasing" singularity profiles and the $\epsilon$ regularity theorems of White [42] give the desired $C^{2}$ estimates.

Two major difficulties remain to be overcome:

(1) Boundary value problem. This was addressed in [37]. More sophisticated barriers that are adapted to the boundary geometry are needed in order to obtain sharper result to cover the area-decreasing case.

(2) Effective estimates in time as $t \rightarrow \infty$. So far, convergence results rely on the sign of the curvature of the ambient space. The $C^{2}$ estimates obtained through blow-up analysis usually deteriorate in time.

In the next section, we present the statements of results which are cleanest when $M_{1}$ and $M_{2}$ are closed Riemannian manifolds with suitable curvature conditions. We remark that there have been several global existence and convergence theorems on higher codimensional graphical mean curvature flows such as $[\mathbf{2 9}, \mathbf{3 0}, \mathbf{3 4}, \mathbf{7}, \mathbf{2}]$, etc. Here we focus on those theorems that have implications on isotopy problems.

\section{Statements of results related to isotopy problems}

3.1. Symplectomorphisms of Riemann surfaces. Let $\left(M_{1}, g_{1}\right)$ and $\left(M_{2}, g_{2}\right)$ be Riemann surfaces with metrics of the same constant curvature. We can normalize so the curvature is $-1,0$ or 1 . Let $f: M_{1} \rightarrow M_{2}$ be an oriented area-preserving map and $\Sigma$ be the graph of $f$ in $M_{1} \times M_{2}$. A oriented area-preserving map is also a symplectomorphism, i.e. $f^{*} \omega_{2}=\omega_{1}$ where $\omega_{1}$ and $\omega_{2}$ are the area forms (or symplectic forms) of $g_{1}$ and $g_{2}$, respectively. The area $A(f)$ of the graph of $f$ is a symmetric function on the symplectomorphism group, i.e. $A(f)=A\left(f^{-1}\right)$ and the mean curvature flow gives a deformation retract of this group to a finite dimensional one.

Theorem 1. ([32, 33, 35], see also $[\mathbf{3 8}])$ Suppose $\Sigma_{0}$ is the graph of a symplectomorphism $f_{0}: M_{1} \rightarrow M_{2}$. The mean curvature flow $\Sigma_{t}$ exists for all $t \in[0, \infty)$ and converges smoothly to a minimal submanifold as $t \rightarrow \infty$. $\Sigma_{t}$ is the graph of a symplectic isotopy $f_{t}$ from $f_{0}$ to a canonical minimal map $f_{\infty}$.

Since any diffeomorphism is isotopic to an area preserving diffeomorphism, this gives a new proof of Smale's theorem [27] that $\mathrm{O}(3)$ is the deformation retract of the diffeomorphism group of $S^{2}$. For a positive genus Riemann surface, this implies the identity component of the diffeomorphism group is contractible.

The result for the positive genus case was also obtained by Smoczyk [29] under an extra angle condition.

In this case, the graph of the symplectomorphism is indeed a Lagrangian submanifolds in the product space. There have been important recent progresses on the Lagrangian minimal surface equation, we refer to the excellent survey article of Brendle $[\mathbf{6}]$ in this direction. 
For an area-decreasing map $f$, i.e. $\left|f^{*} \omega_{2}\right|<\omega_{1}$, the mean curvature flow exists for all time and converges to the graph of a constant map, see [33].

3.2. Area-decreasing maps in higher dimensions. The areadecreasing condition, which turns out to be rather natural for the mean curvature flow, can be generalized to higher dimensions. A Lipschitz map $f: M_{1} \rightarrow M_{2}$ between Riemannian manifolds is area-decreasing if the 2dilation $\left|\Lambda^{2} d f\right|_{p} \mid<1$ for each $p \in M_{1}$. Here $\left.\Lambda^{2} d f\right|_{p}: \Lambda^{2} T_{p} M_{1} \rightarrow \Lambda^{2} T_{f(p)} M_{2}$ is the map induced by the differential $\left.d f\right|_{p}: T_{p} M_{1} \rightarrow T_{f(p)} M_{2}$.

Equivalently, in local orthonormal coordinate systems on the domain and the target, we ask

$$
\left|\frac{\partial f^{\alpha}}{\partial x^{i}} \frac{\partial f^{\beta}}{\partial x^{j}}-\frac{\partial f^{\alpha}}{\partial x^{i}} \frac{\partial f^{\beta}}{\partial x^{j}}\right|<1
$$

for $\alpha \neq \beta, i \neq j$. This is also the same as $H^{2}(f(D)) \leq H^{2}(D)$ for any $D \subset M_{1}$ of finite two-dimensional Hausdorff measure $H^{2}(\cdot)$.

In [31], we proved that area decreasing condition is preserved along the mean curvature flow for the graph of a smooth map $f: S^{n} \rightarrow S^{m}$ between spheres of constant curvature 1. In addition,

Theorem 2. [31] Suppose $n, m \geq 2$. If $f: S^{n} \rightarrow S^{m}$ is an area-deceasing smooth map, the mean curvature flow of the graph of $f$ exists for all time, remains a graph, and converges smoothly to a constant map as $t \rightarrow \infty$.

The most difficult part of the proof is to express the area-decreasing condition as the two-positivity condition (i.e. the sum of the two smallest eigenvalues is positive) for a Lorentzian metric of signature $(n, m)$ and compute the evolution equation of the induced metric.

A simple corollary is the following:

COROllary 3. If $n, m \geq 2$, every area-decreasing map $f: S^{n} \rightarrow S^{m}$ is homotopically trivial.

Gromov [12] shows that for each pair $(n, m)$, there exists a number $\epsilon(n, m)>0$, so that any map from $S^{n}$ to $S^{m}$ with $\left|\Lambda^{2} d f\right|<\epsilon(n, m)$ is homotopically trivial, where $\epsilon(n, m)<<1$. In general, we may consider the $k$-Jacobian $\Lambda^{k} d f: \Lambda^{k} T M_{1} \rightarrow \Lambda^{k} T M_{2}$, whose supreme norm $\left|\Lambda^{k} d f\right|$ is called the $k$-dilation ( $k=1$ is the Lipschitz norm). Guth [13] constructed homotopically non-trivial maps from $S^{n}$ to $S^{m}$ with arbitrarily small 3dilation. It is amazing that 2-dilation is sharp here as it arises naturally from a completely different consideration of the Gauss map of the mean curvature flow (see last section).

3.3. Symplectomorphisms of complex projective spaces. In this section, we consider the generalization of the theorem for symplectomorphisms of Riemann surfaces to higher dimensional manifolds. Let $M_{1}$ and $M_{2}$ be Kähler manifolds equipped with Kähler-Einstein metrics of the same constant scalar curvature. Let $f: M_{1} \rightarrow M_{2}$ be a symplectomorphism. As 
was remarked in the last section, we can consider the graph of $f$ as a Lagrangian submanifold $\Sigma$ in the product space $M_{1} \times M_{2}$ and deform it by the mean curvature flow. A theorem of Smoczyk [28] (see also [25]) implies that the mean curvature flow $\Sigma_{t}$ remains a Lagrangian submanifold. If we can show $\Sigma_{t}$ remains graphical as well, it will corresponds to a symplectic isotopy $f_{t}: M_{1} \rightarrow M_{2}$. The simplest case to be considered in higher dimension is $M_{1}=M_{2}=\mathbb{C P}^{n}$ with the Fubini-Study metric. In a joint work with Medoš, we proved the following pinching theorem.

THEOREM 4. [22] There exists an explicitly computable constant $\Lambda>1$ depending only on $n$, such that any symplectomorphism $f: \mathbb{C P} \mathbb{P}^{n} \rightarrow \mathbb{C P}^{n}$ with

$$
\frac{1}{\Lambda} g \leq f^{*} g \leq \Lambda g
$$

is symplectically isotopic to a biholomorphic isometry of $\mathbb{C P}^{n}$ through the mean curvature flow.

A theorem of Gromov [11] shows that, when $n=2$, the statement holds true without any pinching condition by the method of pseudoholomorphic curves. Our theorem is not strong enough to give an analytic proof of Gromov's theorem for $n=2$. However, for $n \geq 3$, this seems to be the first known result.

Unlike previous theorems, Grassmannian geometry does not quite help here, as the subset that corresponds to biholomorphic isometries does not have any convex neighborhood in the Grassmannian. The integrability condition, or the Gauss-Codazzi equations, is used in an essential way to overcome this difficulty.

\section{References}

[1] B. Andrews, Contraction of convex hypersurfaces in Riemannian spaces. J. Differential Geom. 39 (1994), no. 2, 407-431.

[2] B. Andrews and C. Baker, Mean curvature flow of pinched submanifolds to spheres. J. Differential Geom. 85 (2010), no. 3, 357-395.

[3] E. Bombieri, E. De Giorgi and M. Miranda, Una maggiorazione a priori relativa alle ipersuperfici minimali non parametriche. (Italian) Arch. Rational Mech. Anal. 32 (1969) 255-267.

[4] E. Bombieri, E. De Giorgi, and E. Giusti, Minimal cones and the Bernstein problem. Invent. Math. 7 (1969) 243-68.

[5] K. A. Brakke, The motion of a surface by its mean curvature. Mathematical Notes, 20. Princeton University Press, Princeton, N.J., 1978.

[6] S. Brendle, On the Lagrangian minimal surface equation and related problems. arXiv:1108.0148v1.

[7] J.-Y. Chen, J.-Y. Li, and G. Tian, Two-dimensional graphs moving by mean curvature flow. Acta Math. Sin. (Engl. Ser.) 18 (2002), no. 2, 209-24.

[8] T. H. Colding and W. P. Minicozzi, II, Sharp estimates for mean curvature flow of graphs. J. Reine Angew. Math. 574 (2004), 187-195.

[9] K. Ecker and G. Huisken, Mean curvature evolution of entire graphs. Ann. of Math. (2) 130 (1989), no. 3, 453-471.

[10] K. Ecker and G. Huisken, Interior estimates for hypersurfaces moving by mean curvature. Invent. Math. 105 (1991), no. 3, 547-569. 
[11] M. Gromov, Pseudoholomorphic curves in symplectic manifolds. Invent. Math. 82 (1985), no. 2, 307-347.

[12] M. Gromov, Metric Structures for Riemannian and Non-Riemannian Spaces. Birkhauser, Boston, 1998.

[13] L. Guth, Homotopically non-trivial maps with small k-dilation. Available at http://xxx.lanl.gov/pdf/0709.1241

[14] R. Hamilton, Four-manifolds with positive curvature operator. J. Differential Geom. 24 (1986), no. 2, 153-179.

[15] G. Huisken, Asymptotic behavior for singularities of the mean curvature flow. J. Differential Geom. 31 (1990), no. 1, 285-299, MR1030675, Zbl 0694.53005.

[16] G. Huisken, Flow by mean curvature of convex surfaces into spheres. J. Differential Geom. 20 (1984), no. 1, 237-266.

[17] G. Huisken and C. Sinestrari, Mean curvature flow singularities for mean convex surfaces. Calc. Var. Partial Differential Equations 8 (1999), no. 1, 1-14.

[18] G. Huisken and C. Sinestrari, Convexity estimates for mean curvature flow and singularities of mean convex surfaces. Acta Math. 183 (1999), no. 1, 45-70.

[19] T. Ilmanen, Elliptic regularization and partial regularity for motion by mean curvature. Mem. Amer. Math. Soc. 108 (1994), no. 520.

[20] H. Jenkins and J. Serrin, The Dirichlet problem for the minimal surface equation in higher dimensions. J. Reine Angew. Math. 229 (1968) 170-87.

[21] H. B. Lawson and R. Osserman, Non-existence, non-uniqueness and irregularity of solutions to the minimal surface system. Acta Math. 139 (1977), no. 1-2, 1-17.

[22] I. Medoš and M.-T. Wang, Deforming symplectomorphisms of complex projective spaces by the mean curvature flow. J. Differential Geom. 87 (2011), no. 2, 309-342.

[23] C. B. Morrey, Jr. Multiple integrals in the calculus of variations. Die Grundlehren der mathematischen Wissenschaften, Band 130 Springer-Verlag New York, Inc., New York 1966.

[24] J. Moser, A new proof of De Giorgi's theorem concerning the regularity problem for elliptic differential equations. Comm. Pure Appl. Math. 13 (1960) 457-68.

[25] Y.-G. Oh, Mean curvature vector and symplectic topology of Lagrangian submanifolds in Einstein-Kähler manifolds. Math. Z. 216 (1994), no. 3, 471-482.

[26] R. Osserman, A survey of minimal surfaces. Second edition. Dover Publications, Inc., New York, 1986.

[27] S. Smale, Diffeomorphisms of the 2-sphere. Proc. Amer. Math. Soc. 10 1959, 621-626.

[28] K. Smoczyk, A canonical way to deform a Lagrangian submanifold. preprint, dgga/9605005.

[29] K. Smoczyk, Angle theorems for the Lagrangian mean curvature flow. Math. Z. 240 (2002), no. 4, 849-883.

[30] K. Smoczyk and M.-T. Wang, Mean curvature flows of Lagrangian submanifolds with convex potentials. J. Differential Geom. 62 (2002), no. 2, 243-257.

[31] M.-P. Tsui and M.-T. Wang, Mean curvature flows and isotopy of maps between spheres. Comm. Pure. Appl. Math. 57 (2004), no. 8 , 1110-1126.

[32] M.-T. Wang, Deforming area preserving diffeomorphism of surfaces by mean curvature flow. Math. Res. Lett. 8 (2001), no.5-6, 651-662.

[33] M.-T. Wang, Mean curvature flow of surfaces in Einstein Four-Manifolds. J. Differential Geom. 57 (2001), no.2, 301-338.

[34] M.-T. Wang, Long-time existence and convergence of graphic mean curvature flow in arbitrary codimension. Invent. math. 148 (2002) 3, 525-543.

[35] M.-T. Wang, A convergence result of the Lagrangian mean curvature flow. Third International Congress of Chinese Mathematicians. Part 1, 2, 291-295, AMS/IP Stud. Adv. Math., 42, pt. 1, 2, Amer. Math. Soc., Providence, RI, 2008.

[36] M.-T. Wang, Gauss maps of the mean curvature flow. Math. Res. Lett. 10 (2003), no. 2-3, 287-299. 
[37] M.-T. Wang, Lectures on mean curvature flows in higher codimensions. Handbook of geometric analysis. No. 1, 525-543, Adv. Lect. Math. (ALM), 7, Int. Press, Somerville, MA, 2008.

[38] M.-T. Wang, Some recent developments in Lagrangian mean curvature flows. Surveys in differential geometry. Vol. XII. Geometric flows, 333-347, Surv. Differ. Geom., 12, Int. Press, Somerville, MA, 2008.

[39] M.-T. Wang, The Dirichlet problem for the minimal surface system in arbitrary codimension. Comm. Pure. Appl. Math. 57 (2004), no. 2, 267-281.

[40] X.-J. Wang, Convex solutions to the mean curvature flow. Ann. of Math. (2) 173 (2011), no. 3, 1185-1239.

[41] B. White, The nature of singularities in mean curvature flow of mean-convex sets. J. Amer. Math. Soc. 16 (2003), no. 1, 123-138.

[42] B. White, A local regularity theorem for classical mean curvature flow. Ann. of Math. (2) 161 (2005), no. 3, 1487-1519, MR2180405, Zbl 1091.53045.

Department of Mathematics, Columbia University, 2990 Broadway, New YORK, NY 10027, USA 